\title{
Rearch on Electromagnetic Scattering from ocean covered by foams
}

\author{
GAO Qian ${ }^{1}$, YUE Hongwei ${ }^{1}$,WANG Zhenxin ${ }^{1}$, JI Yajun ${ }^{1}$,LI Ximin ${ }^{2}$ \\ ${ }^{1}$ Air Force Communication NCO Academy, Dalian, China \\ ${ }^{2}$ Air and Missile Defense College,Air Force Engineering University,Xi'an,China
}

\begin{abstract}
Back scattering of ocean surface is investigated by considering the foams coverage on its surface. Firstly, by adopting modified two-scale mode and the vector radiative transfer theory, zero and first order scattering coefficients are obtained by solving VRT equations. Then, MIE theory is used to get the scattering from foams, and fraction of foams coverage is analyzed by changing parameters of wind speed and temperature difference of ocean-air. Finally, the modified two-scale model and algorithms are verified by comparing its results with measured results, and simulations of calculating back scattering from ocean surface with and without foams are carried out. The conclusion is obtained that wind speed has different influence on back scattering under different incident angles.
\end{abstract}

\section{INTRODUCTION}

In natural world, the ocean surface will be partly covered by foams when the wind speed above the ocean reaches a certain extent. For example, one third of the ocean surface will be covered by foams as the wind speed is $25 \mathrm{~m} / \mathrm{s}$. Therefore, ocean surface scattering and foams particle volume scattering must be considered both in order to get the accurate calculation results, when the ocean scattering characteristic is analyzed. Manahan treated ocean foams as water-air bubbles, instead of sphere water partical in his research ${ }^{[1]}$.Y.Q. Jin investigated the EM scattering characteristic of ocean covered by foams firstly by combing vector radiative transfer theory (VRT) and two-scale model ${ }^{[2]}$. In this paper, the modified two-scale model, the vector radiative transfer theory, and MIE theory are adopted to analyze the EM scattering from ocean surface covered by foams.

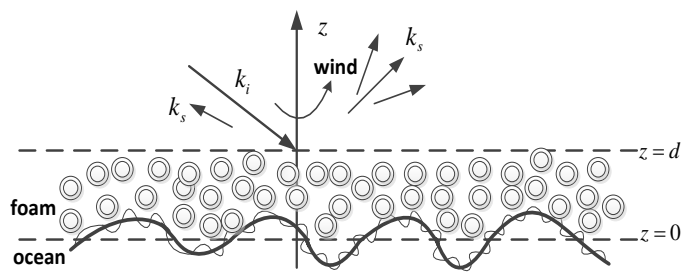

Fig.1 Two-scale ocean surface covered by foams

\section{VECTOR RADIATIVE TRANSFER EQUATION OF OCEAN SURFCE CONSIST OF FOAMS}

In Fig.1, the vector radiation transfer equations ${ }^{[3]}$ of foam layers can be writed as,

$$
\begin{aligned}
& \cos \theta_{s} \frac{d}{d z} \bar{I}\left(\theta_{s}, \phi_{s}, z\right)=-\overline{\bar{K}}_{e}\left(\theta_{s}, \varphi_{s}\right) \cdot \bar{I}\left(\theta_{s}, \phi_{s}, z\right)+\int_{0}^{2 \pi} d \phi^{\prime} \int_{0}^{\pi / 2} d \theta^{\prime} \sin \theta_{s} \\
& \cdot \stackrel{=}{P}\left(\theta_{s}, \phi_{s} ; \theta^{\prime}, \phi^{\prime}\right) \bar{I}\left(\theta^{\prime}, \phi^{\prime}, z\right)+\overline{\mathrm{Q}}\left(z, \theta_{s}, \phi_{s}\right) \\
& -\cos \theta_{s} \frac{d}{d z} \bar{I}\left(\pi-\theta_{s}, \phi_{s}, z\right)=-\overline{\bar{K}}_{e}\left(\theta_{s}, \varphi_{s}\right) \cdot \bar{I}\left(\pi-\theta_{s}, \phi_{s}, z\right) \\
& +\int_{0}^{2 \pi} d \phi^{\prime} \int_{0}^{\pi / 2} d \theta^{\prime} \sin \theta_{s} \cdot \stackrel{=}{P}\left(\pi-\theta_{s}, \phi_{s} ; \theta^{\prime}, \phi^{\prime}\right) \cdot \bar{I}\left(\theta^{\prime}, \phi^{\prime}, z\right) \\
& +\int_{0}^{2 \pi} d \phi^{\prime} \int_{0}^{\pi / 2} d \theta^{\prime} \sin \theta_{s} \cdot \stackrel{=}{P}\left(\pi-\theta_{s}, \phi_{s} ; \pi-\theta^{\prime}, \phi^{\prime}\right) \\
& \cdot \bar{I}\left(\pi-\theta^{\prime}, \phi^{\prime}, z\right)+\overline{\mathrm{Q}}\left(z, \theta_{s}, \phi_{s}\right)
\end{aligned}
$$

In formula (1), $\theta^{\prime}, \phi^{\prime}, \theta_{s}, \phi_{s}$ represents incident vertical angle, incident horizontal angle, scattering vertical angle and scattering horizonal angle respectively, $I(\theta, \varphi, z)$ and $K_{e}$ represents $4 \times 1$ column vetor and extinction matrix respectively, $\overline{\mathrm{Q}}$ and $\stackrel{=}{P}\left(\theta, \phi ; \theta^{\prime}, \phi^{\prime}\right)$ represents source of heat and phase matrix respectively. Partical satisfied Rayleigh approximation, so the phase

* Corresponding author: gaoqian717@163.com 
matirx $\stackrel{=}{P}\left(\theta, \phi ; \theta^{\prime}, \phi^{\prime}\right)$ can be obtained from Rayleigh approxiamtion.

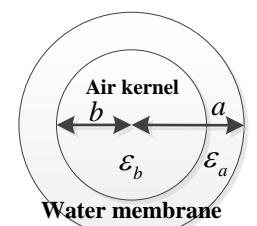

Fig.2 Geometric structure of foam

In physical practice, the foams resulted by wind speed may be equal to several bubble partical. Foam partical can be described as the structure model in Fig.2 which obeys Gama distribution ${ }^{[4]}$. In Fig. $2, a=250 \mu \mathrm{m}$ represents the outer radius of foam partical, $b=230 \mu \mathrm{m}$ represents the inner radius of foam partical, then the thickness of foam partical membrane can be obtained as $a-b=20 \mu \mathrm{m}$. The thickness of ocean foams layer is set to be $2 \mathrm{~cm}$ in the following simulation . The basitic scattering coefficient of ocean surface contained foams can be written as ${ }^{[3]}$

$$
\sigma_{p q}\left(\theta_{s}, \phi_{s} ; \theta_{i}, \phi_{i}\right)=\frac{4 \pi \cos \theta_{s} I_{p}\left(\theta_{s}, \phi_{s}\right)}{I_{q}\left(\theta_{i}, \phi_{i}\right)}
$$

In formula (3), $\theta_{i}, \varphi_{i}$ represents the incident vertiacal angle and horizontal angle respectively, $I_{q}, I_{p}$ represents incident spectra and scattering spectra respectively。 Scattering from single foam can be calculated through Mie theory, then VRT (Vector Radiation Transmission) differential-infergral equations are obtained by method of constant variation and boundary conditions. The solution of VRT equations is solved by using iteration method. Finally, zero order and first order scattering coefficients of ocean surface covered by poams are obtained accordingly. $\sigma_{p q(\mathrm{a})}^{(0)}$ represents zero order scattering coefficient, $\sigma_{p q(\mathrm{~b})}^{(1)}, \sigma_{p q(\mathrm{c})}^{(1)}, \sigma_{p q(\mathrm{~d})}^{(1)}$ and $\sigma_{p q(\mathrm{e})}^{(1)}$ represent first order scattering coefficients. Their formulas can be written as follow ${ }^{[4]}$ :

(1) Scattering only scattering from two-scale ocean surface

$$
\sigma_{p q(\mathrm{a})}^{(0)}\left(\theta_{s}, \phi_{s}, \theta_{i}, \phi_{i}\right)=\sigma_{p q} e^{-k_{e} d\left(1 / \cos \theta_{s}+1 / \cos \theta_{i}\right)}
$$

(2) Scattering only scattering from water bubble partical

$$
\sigma_{p q(b)}^{(1)}\left(\theta_{s}, \phi_{s}, \theta_{i}, \phi_{i}\right)=\frac{4 \pi}{k_{e}} \cos \hat{}_{s} \bar{P}_{p q}\left(\theta_{s}, \phi_{s}, \pi-\theta_{i}, \phi_{i}\right)\left(1-e^{-k_{e} d\left(1 / \cos \theta_{s}+1 / \cos \theta_{i}\right)}\right)(5)
$$

(3) Scattering firstly from water bubble partical then from rough surface

$$
\begin{aligned}
& \sigma_{p q(\mathrm{c})}^{(1)}\left(\theta_{s}, \phi_{s}, \theta_{i}, \phi_{i}\right)=\frac{4 \pi}{k_{e}} \cos \theta_{s} \int_{0}^{2 \pi} d \theta^{\prime} \sin \theta^{\prime} \int_{0}^{2 \pi} d \phi^{\prime} \sum_{l=v, h} \overline{\bar{P}}_{p l}\left(\theta_{s}, \phi_{s}, \theta^{\prime}, \phi^{\prime}\right) \\
& ={ }_{p q}\left(\theta^{\prime}, \phi^{\prime}, \pi-\theta_{i}, \phi_{i}\right) \times \frac{\cos \theta^{\prime}}{\cos \theta_{s}-\cos \theta^{\prime}} e^{-k_{e} d\left(1 / \cos \theta^{\prime}-1 / \cos \theta_{s}\right)}(6)
\end{aligned}
$$

(4) Scattering firstly from two-scale ocean surface then from water bubble partical

$$
\begin{aligned}
& \sigma_{p q(\mathrm{~d})}^{(1)}\left(\theta_{s}, \phi_{s}, \theta_{i}, \phi_{i}\right)=\frac{4 \pi}{k_{e}} \cos \theta_{s} \int_{0}^{\pi / 2} d \theta^{\prime} \sin \theta^{\prime} \int_{0}^{2 \pi} d \phi^{\prime} \sum_{m=v, h} \overline{\bar{R}}_{p m}\left(\theta_{s}, \phi_{s}, \pi-\theta^{\prime}, \phi^{\prime}\right) \\
& \times \overline{\bar{P}}_{m q}\left(\pi-\theta^{\prime}, \phi^{\prime}, \pi-\theta_{i}, \phi_{i}\right) \times \frac{\cos \theta_{i}}{\cos \theta_{i}-\cos \theta^{\prime}} e^{-k_{e} d\left(1 / \cos \theta^{\prime}-1 / \cos \theta_{i}\right)}(7)
\end{aligned}
$$

(5) Scattering firstly from two-scale ocean surface then from water bubble partical, finally from two-scale ocean rough surface

$$
\begin{gathered}
\sigma_{p q(\mathrm{e})}^{(1)}\left(\theta_{s}, \phi_{s}, \theta_{i}, \phi_{i}\right)=\frac{4 \pi}{k_{e}} \cos \theta_{s} \int_{0}^{\pi / 2} d \theta^{\prime} \sin \theta^{\prime} \int_{0}^{2 \pi} d \phi^{\prime} \sum_{l=v, h} \overline{\bar{R}}_{p l}\left(\theta_{s}, \phi_{s}, \pi-\theta^{\prime}, \phi^{\prime}\right) \\
\times \int_{0}^{\pi / 2} d \theta^{\prime \prime} \sin \theta^{\prime \prime} \int_{0}^{2 \pi} d \phi^{\prime \prime} \times \sum_{m=v, h} \overline{\bar{P}}_{l m}\left(\pi-\theta^{\prime}, \phi^{\prime}, \theta^{\prime \prime}, \phi^{\prime \prime}\right) \overline{\bar{R}}_{m q}\left(\theta^{\prime \prime}, \phi^{\prime \prime}, \theta_{i}, \phi_{i}\right) \\
\times \frac{\cos \theta^{\prime \prime}}{\cos \theta^{\prime}+\cos \theta^{\prime \prime}}\left(1-e^{-k_{e} d\left(1 / \cos \theta^{\prime}+1 / \cos \theta^{\prime \prime}\right)}\right) e^{-k_{e} d\left(1 / \cos \theta_{i}+1 / \cos \theta_{s}\right)}(
\end{gathered}
$$

Where $K_{e}$ is extinction coefficient, $\sigma_{p q}$ can be obtained by two-scale model。 Physical meanings of the coefficeints above are demonstrated in Fig. 3 (a) (e).

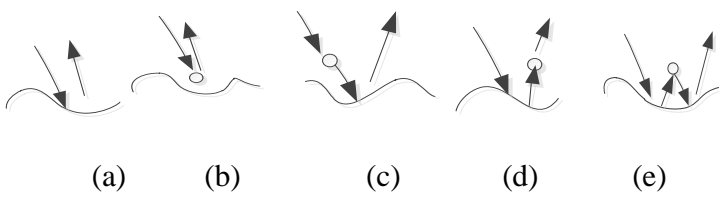

Fig.3 Zeror and first order solution of the scattering

In conclusion, the scattering coefficients of ocean surface covered by foams are written as

$$
\begin{array}{r}
\sigma_{p q}^{\mathrm{foam}}\left(\theta_{s}, \phi_{s}, \theta_{i}, \phi_{i}\right)=\sigma_{p q(\mathrm{a})}^{(0)}\left(\theta_{s}, \phi_{s}, \theta_{i}, \phi_{i}\right)+\sigma_{p q(\mathrm{~b})}^{(1)}\left(\theta_{s}, \phi_{s}, \theta_{i}, \phi_{i}\right) \\
+\sigma_{p q(\mathrm{c})}^{(1)}\left(\theta_{s}, \phi_{s}, \theta_{i}, \phi_{i}\right)+\sigma_{p q(\mathrm{~d})}^{(1)}\left(\theta_{s}, \phi_{s}, \theta_{i}, \phi_{i}\right)+\sigma_{p q(\mathrm{e})}^{(1)}\left(\theta_{s}, \phi_{s}, \theta_{i}, \phi_{i}\right)
\end{array}
$$

\section{SCATTERING AND EXTINCTION COEFFICIENTS OF FOAMS}

MIE theroy can be adopted to analyze scattering from sphere hollow partical in Fig.2, $\mathrm{T}$ matrix ${ }^{[5]}$ of MIE scattering can be written as.

$$
\begin{aligned}
& T_{n}^{(M)}=-\frac{\left[\delta j_{n}(\delta)\right]^{\prime}\left[j_{n}(\varsigma)+B_{n}(\zeta, \chi) y_{n}(\varsigma)\right]-\left\{\left[\varsigma j_{n}(\varsigma)\right]^{\prime}+B_{n}(\zeta, \chi)\left[\varsigma y_{n}(\varsigma)\right]^{\prime}\right\} j_{n}(\delta)}{\left[\delta h_{n}(\delta)\right]^{\prime}\left[j_{n}(\varsigma)+B_{n}(\zeta, \chi) y_{n}(\varsigma)\right]-\left\{\left[\varsigma j_{n}(\varsigma)\right]^{\prime}+B_{n}(\zeta, \chi)\left[\varsigma y_{n}(\varsigma)\right]^{\prime}\right\} h_{n}(\delta)} \\
& T_{n}^{(N)}=-\frac{\left[\delta j_{n}(\delta)\right]^{\prime} \varsigma\left[j_{n}(\varsigma)+A_{n}(\zeta, \chi) y_{n}(\varsigma)\right]-\left\{\left[\varsigma j_{n}(\varsigma)\right]^{\prime}+A_{n}(\zeta, \chi)\left[\varsigma y_{n}(\varsigma)\right]^{\prime}\right\} \delta^{2} j_{n}(\delta)}{\left[\delta h_{n}(\delta) \zeta^{\prime} \varsigma^{2}\left[j_{n}(\varsigma)+B_{n}(\zeta, \chi) y_{n}(\varsigma)\right]-\left\{\left[\zeta j_{n}(\varsigma)\right)^{\prime}+B_{n}(\zeta, \chi)\left[\zeta y_{n}(\zeta)\right)^{\prime}\right\} h_{n}(\delta)\right.}
\end{aligned}
$$

Where $j_{n}$ is spherical Bessel function, $h_{n}$ is spherical Hankel function, $y_{n}$ is Neumann functions, $\delta=k a, \varsigma=k_{a} a, \zeta=k_{b} b, \chi=k_{b} a, a$ is the outer radius of the partical,$b$ is the inner radius of the partical, $k$ is the wavenumber of incident wave in free space, $k_{b}$ is wavenumber in nucleus, $k_{a}$ is wavenumber in shell. And extinction cross and cross section can be written as beblow,

$$
Q_{e x t}=\frac{2}{(k a)^{2}} \sum_{n=1}^{\infty}(2 n+1) \operatorname{Re}\left(-T_{n}^{(N)}-T_{n}^{(M)}\right)
$$




$$
Q_{s c a}=\frac{2}{(k a)^{2}} \sum_{n=1}^{\infty}(2 n+1)\left(\left|-T_{n}^{(N)}\right|^{2}+\left|-T_{n}^{(M)}\right|^{2}\right)
$$

Then the formulas of extinction coefficient and scattering coefficient of foams are obtained as below ${ }^{[6]}$,

$$
\begin{gathered}
K_{e}=N_{0} \frac{2 \pi}{k^{2}} \sum_{n=1}^{\infty}(2 n+1) \operatorname{Re}\left(-T_{n}^{(N)}-T_{n}^{(M)}\right) \\
K_{s}=N_{0} \frac{2 \pi}{k^{2}} \sum_{n=1}^{\infty}(2 n+1)\left(\left|-T_{n}^{(N)}\right|^{2}+\left|-T_{n}^{(M)}\right|^{2}\right)
\end{gathered}
$$

where $N_{0}$ represents foams numbers in unit volume, and it can be calculated by volume intergration.

\section{ANALYSIS OF FRACTION OF FAOMS COVERAGE}

Fraction of foams coverage on ocean surface driven by wind, is closely related to wind speed,temperature of seawater. Before accurately analyzing scattering property of different ocean zone covered by foams, fraction of foams coverage on ocean surface must be analyzed firstly. Under stable atmosphere conditions, fraction of foams coverrage on ocean surface may be solved by the following formula ${ }^{[7]}$,

$$
C=2.32 \times 10^{-6} U_{10}^{3.4988}
$$

Otherwise, when aimoshpere is in unstable conditions( $2^{\circ} \mathrm{C}<$ temperature difference of oceanair $<12^{\circ} \mathrm{C}$ ), the formula of fraction of foams coverrage is

$$
C=0.43 \times 10^{-6} U_{10}^{3.6842}
$$

Only the correlation between fraction of foams coverrage and wind speed is reflected in formula (17). In order to anayze how temperature difference of ocean-air influences fraction of foams coverrage,the following model is adopted,

$$
C=1.95 \times 10^{-5} U_{10}^{2.55} \exp (0.0861 \Delta T)
$$

Where $\Delta T$ represents temperature difference of ocean-air, $U_{10}$ represents wind speed 10 meters abvove ocean surface. Fig.4 shows the correlations between fraction of foams coverrage and wind speed, temperature difference of ocean-air. As the wind speed and temperature difference increase, fraction of foams coverrage is increasing. And the following simualtions of fraction of foams coverage are done by formula (16) under the conditions of moderate temperature difference and stable atmoshpere.

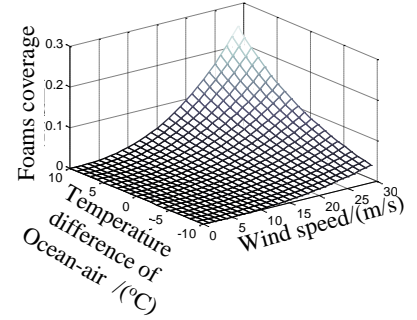

Fig.4 Relations of foams coverage - temperature difference of ocean-air and wind speed

The scatttering coefficients of two-scale ocean surface considering foams influence can be written as,

$$
\sigma_{p q}^{\text {all }}=(1-C) \sigma_{p q}+C \sigma_{p q}^{\text {foam }}
$$

\section{CALCULATION SIMULATION AND ANALYSIS}

Before calculating the EM scattering from ocean surface containing foams with the modified two-scale model, the accuracy of algorithm must be validated. The hhpolarized back scattering codfficient of two-scale gause rough ocean surface is calculated by adopting the modified two-scale model, and the results are validated by comparing with measured datas of literature ${ }^{[7]}$ in Fig.5. In the simulation, requency of incident wave is $8.93 \mathrm{GHz}$, in Gaussian spectral, parameters $m, k h, k l$ are obtained

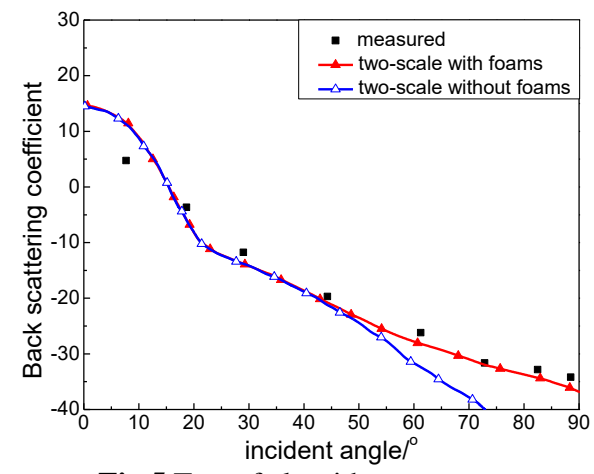

Fig.5 Test of algorithm accuracy

by converting the ocean spectral with wind speed of 14 16 $\mathrm{n}$ mile/hour into Gaussian spectral,and $h$ represents rms height of rough surface, $l$ represents correlation length, $m$ represents rms fraction of foams coverrage, the temperature of ocean surface is set to be $17.4^{\circ} \mathrm{C}$ which is the global average seawater temperature. The salinity of seawater is set to be $32.54 \%$ as the global average salinity. And the dielectric permittivity of seawater is calculated as (57.63,37.73). By analyzing results in Fig.5, the difference is slight by comparing foams and no-foams, and both are conincide well with the measured results. But under increasing incident angle, the decay of twoscale results becomes larger. The decay trend can be effectively weakened under large intendance angle, and the calculating results conincide with measured data better. Another thesis from Fig. 5 is that back scattering coefficent of ocean surface is decreasing as the incident angle increasing. 


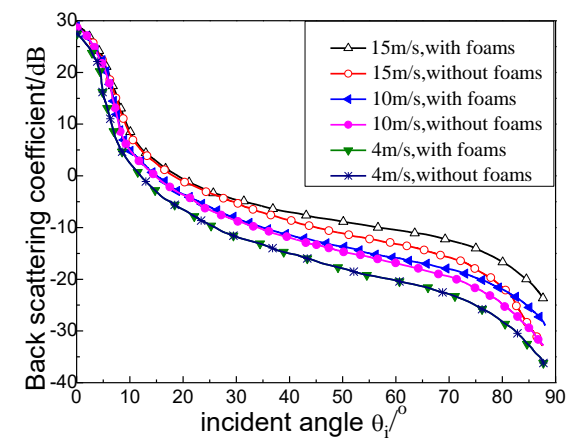

Fig.6 Back sacttering coefficients of ocean surface under differnent wind speed

Then, the modified two-scale model is adopted to get the back scattering property of oceans surface generated by E- spectral functions, and the influence of wind speed to back scattering coeffient is specially considered. The simulation results is shown in Fig.6, under $\mathrm{Ku}$ frequency band with VV polarization, the dielectric permittivity of seawater is calculated as $(42.08,39.46)$ by the same model used in the previouse example in Fig.5. It is indicated that foams coverage and wind speed have slight influence on the back scattering under the smaller incident angle. While, under large incident angle, the back scattering of ocean surface is increasing as wind speed increasing. When wind speed is $4 \mathrm{~m} / \mathrm{s}$, foams coverge has un-obvious influence on back scattering. And as wind speed increasing, the differecne of with and without foams bacomes more and more obviouse. This is explained by fraction of foams coverage bacomes larger as wind speed increasing.

\section{CONCLUSION}

Through the investigation of calculating back scattering from ocean surface covered by foams, we can concluded that back scattering coefficients of ocean with and without foams have significant difference, and under larger incident angle, greater wind speed caused larger back scattering coefficient.

\section{REFERENCES}

1. W Manahan E C, MacNiocaill G. Oceanic Whitecaps and Their Role in Air-Sea Exchange Process[M]. Boston: D.Reidel Publishing, 1986:113123.

2. Y.-Q. Jin. Remote sensing theory of electromagnetic scattering and thermal emission $[\mathrm{M}]$. Beijing: Science Press, 1993.

3. Y.-Q. Jin. Some results from the radiative wave equation for a slab of randomly, densely distributed scatters[J]. J.Quant. Spectrosc. and Radiat. Transfer, 1988, 39 (2): 83-98.

4. Villarino R, Camps A, Vall-Ilossera M, et al. Sea foam effects on the brightness temperature at Lband[C]. IEEE International Geoscience and Remote Sensing Symposium, 2003: 3076-3078.
5. Z. S. Wu, Y. P. Wang. Electromagnetic scattering for multilayered sphere: recursive algorithms[J]. Radio Sci., 1991, 26 (6): 1393-1401.

6. Droppleman J D. Apparent microwave emissivity of sea foam[J]. Journal of Geophysical Research. 1970, 75(3): 696-698.

7. Liang Yu, Guo L.-X. Study of the electromagnetic scattering from the rough sea surface with bubbles/foams by the modified two-scale method[J]. Acta Physical Sinica, 2009, 58(9): 6158-6166. 